\title{
Xenotransplantation - The Donor Welfare Perspective
}

\author{
By Svein Aage Christoffersen
}

Faculty of Theology, University of Oslo, P.O.Box 1023 Blindern, NO-0315 Oslo, Norway. Tel: +4722850320, E-mail: s.a.christoffersen@teologi.uio.no

\section{Introduction}

In this lecture xenotransplantation is considered from the point of view of animal ethics. The main point is to show that a specific cluster of arguments commonly used in favour of xenotransplantation, simplifies the ethical questions involved. This simplification may be favourable for political reasons in a short time perspective, but may at the same time be disastrous from an ethical point of view in the long run. If the ethical questions regarding xenotransplantation are reduced to a simple yes or no, this will overshadow more important questions of how xenotransplantation may be implemented in an ethically responsible way. Resisting this simplification, we have to explore instead the necessary conditions under which xenotransplantation may be carried through or rejected.

Ethics is often expected to deliver simple answers to complex questions. The bottom line of an ethical argument is expected to say yes or no. This way of understanding ethical contributions to the public and scientific discourse about xenotransplantation, or any scientific and public discourse, is however not in accordance with the ethical point of view. People who expect simple and clear-cut answers to moral questions do not - or at least not always - expect these unambiguous answers because they want ethics to be of importance to the questions involved. Very often they expect unambiguous answers for the opposite reason. They want clear-cut an- swers just to get rid of ethical considerations. When ethics is reduced to a simple yes or no, then it is of no use and not worth taking into consideration. So ethics is from this point of view trapped in a "catch 22 ". If it can't deliver simple answers, it is of no use, and if it delivers simple answers, it certainly is of no use.

Contrary to the demand for simplicity I therefore want to say, using the words of the poet Søren Kierkegaard, "that in this day and age when everybody is striving to make everything more easy, my task is to make everything more difficult", or at least as difficult as possible within twenty minutes. I am not doing this because I love difficulties, but because I think we have to consider more deeply what xenotransplantation is all about. Putting something into action, there is a world of difference between doing it with or without an extended consciousness of what we are actually doing. So let us look more closely into the matter of xenotransplantation from the perspective of animal ethics.

\section{Animal welfare and xenotransplantation}

The main arguments in favour of breeding genetically modified pigs for xenotransplantation are summed up in the following statement from the 1996 Nuffield Council Report (1) on the ethics of xenotransplantation: 


\begin{abstract}
"While the pig is an animal of sufficient intelligence and sociability to make welfare considerations paramount, there is less evidence that it shares capacities with human beings to the extent that primates do. As such, the adverse effects suffered by the pigs used to supply organs for xenotransplantation would not outweigh the potential benefits to human beings. It is also difficult to see how, in a society in which the breeding of pigs for food and clothing is accepted, their use for life-saving medical procedures such as xenotransplantation could be unacceptable."
\end{abstract}

From this point of view xenotransplantation seems to be a clear-cut case. Of course, taken literally the Nuffield Council is not rejecting animal welfare considerations. On the contrary, the statement says that animal welfare perspectives are paramount. But put into practice it says that animal welfare perspectives may not be given preference when at variance with human interests. Or put more bluntly: we may put weight on animal welfare, provided this does not jeopardize human welfare. But considering animal welfare only when it is consistent with human welfare is next to nothing. The problem is what we ought do when animal welfare is not consistent with human welfare. And in these situations, the Council says, animal welfare has to make way for human welfare.

Reading the statement more closely we may identify three main arguments for this point of view. Firstly: Xenotransplantation has to do with the saving of human lives, and when human life has to be weighted against animal life, then human life takes priority. Today people are dying because of the shortage of organs available for transplantation, so breeding and killing animals for xenotransplantation will compensate for this shortage and therefore save the lives of human beings. In this situation, forced to choose between animal lives and human lives, human lives have the preference.

Secondly: From a biological point of view we may prefer to use organs taken from primates for xenotransplantation. But from an ethical point of view primates are too close to human beings. They may react and suffer much in the same way as human beings do. Restrictions put on the use of human transplant may therefore apply also to the use of transplants taken from non-human primates. Pigs, on the other hand, are different from human beings to an extent that primates are not; hence, arguments stated against the use of primates for xenotransplantation are not valid with regard to pigs.

Thirdly: The use of animals is not at all extraordinary. The use of pigs for human benefits is established long ago by the breeding of pigs for food and clothing. When this is so, the breeding of pigs for xenotransplantation may be just as, or even more, legitimate.

Let us consider these arguments more closely, one by one, beginning with the second argument. What this argument says, is not just that the arguments against the use of primates are not applicable to the use of pigs. It also implies that the arguments against the use of primates are conclusive. This is why primates are not in use for xenotransplantation. But why are these arguments not conclusive as far as pigs are concerned? Of course there are differences between pigs and primates, but are these differences significant? Pigs may not possess the same intelligence and sociability as primates; let us take that for granted, at least for the sake of argument. Does this imply by logic that pigs do not have the same capacity for suffering, or that the suffering brought upon pigs does not count because they are not as smart and intelligent as primates are? Is the difference between primates and pigs lager than the difference between primates and human beings, regarding intelligence and sociability? 
The question of suffering is a utilitarian one. But utilitarianism is not the only perspective relevant in this case. It is also possible or even necessary to apply a deontological perspective and ask whether a pig has any right to lead a pig's way of life. A pig is not just an isolated organism capable of responding to external stimuli. To be a pig is also to lead a pig's way of life. The word "rights" is, as you all know, controversial in animal ethics. My point of view may be phrased like this: Regardless of suffering I think we have good reasons for saying that pigs have a right to lead a pig's way of life. But if the word "right" is offending you, I may just as well say that we have a certain obligation to permit pigs to lead a pig's way of life. This is not controversial with regard to primates, so why should it be controversial with regard to pigs? If we are not convinced by the reasons for treating pigs and primates differently, conclusions may be drawn in two different directions. We may either lower the primates down to the pig's level, permitting the use of primates for xenotransplantation too, or we may raise the pigs to a higher level and say that pigs are to be protected against xenotransplantation to the same extent that primates are.

The third argument says, as we have heard already, that the use of pigs for xenotransplantation is made legitimate by the extensive use of pigs for food and clothing. But this argument does not hold water either. There are several important differences between the breeding of pigs for xenotransplantation and the breeding of pigs for food and clothing, and because of these differences it is impossible to make xenotransplantation acceptable by just pointing to our use of animals for food and clothing. Let me mention very briefly just three of these reasons.

The first reason is that the use of pigs for xenotransplantation requires extensive use of animal experimentation. It is not controversial, I think, to say that these experiments have caused extensive animal suffering, and that they are likely to cause animal suffering in the future, too. But this is not the main point in my argument, because the point is that these experiments are not within the limits of what a farmer is allowed to do to his animals. There is a basic difference between a farmer and a researcher, and if we take into consideration how the researcher is allowed to treat an animal, this is an exception to ordinary requirements regarding farm animals. So the ordinary use of pigs for food and clothing is not sufficient to make animal experimentation acceptable.

The second reason is that the use of pigs for xenotransplantation requires transgenic pigs and hence genetic transformation of the pigs in use. But when we breed pigs for food and clothing, genetic engineering is not permitted. This has of course partly to do with food safety, but that is not all there is to it. The prohibition of genetically modified farm animals is also meant to protect animals against infringement and injustice. So breeding pigs for food and clothing does not make genetic engineering legitimate. The third reason is that the way of breeding pigs for xenotransplantation is not in concordance with rules normally applied to the keeping and confinement of farm animals. Especially because of the danger of infection, pigs in use for xenotransplantation may be kept isolated to an extent far above what is normally accepted for farm animals.

These and similar reasons taken together explain why the use of pigs for food and clothing does not make the use of pigs for xenotransplantation acceptable. This does not mean of course that xenotransplantation is to be rejected. The point is that eating pork and wearing a fur coat are as such not sufficient to legitimate xenotransplantation.

These lines of argument lead to the conclusion that just playing human life off against animal 
life is a superficial simplification. Giving human life a preference over and against animal life does not imply a carte blanche to treat animals according to our own convenience only. Moreover, just playing human life off against animal life implies that we have no other alternative. Is that true? If we resign from xenotransplantation does that imply that we have to resign from transplantation all together? Of course it doesn't mean that. The shortage of human organs may also be compensated for by our efforts to increase the availability of human organs. One may of course be pessimistic regarding the possibility of satisfying the demand for organs by these efforts, but xenotransplantation is no secure road to success either. And then we have the use of stem cells. In the end the situation may very well be that xenotransplantation appears to be a sidetrack. This may take some years of course, but there are nevertheless reasons to believe, that the main track to organ supplement would be stem cell research. The question of xenotransplantation is therefore not a question of saving human lives or not, but a question of different roads to the saving of human lives.

\section{The relationship between humans and pigs}

Another important question is how, or to what extent, xenotransplantation may affect our understanding of the relationship between animals and human beings. Usually this question has been discussed with regard to the human selfesteem, self-consciousness and self-understanding. My perspective is however, the donor perspective, so my question is if or to what extent xenotransplantation may change our understanding of animals.

Some have argued that xenotransplantation will pave the way to a more inclusive understanding of animals. But this is just wishful thinking. As mentioned already, using pigs for xenotransplantation presupposes the difference between pigs and humans. Blurring this difference means making xenotransplantation more dubious. I think we have a real dilemma here. On the one hand pigs may be used for xenotransplantation because they have a biological resemblance to human beings. On the other hand pigs may be used for xenotransplantation just because they are not human beings, or not even primates. The closer pigs are connected to human beings from an ethical point of view, the more dubious xenotransplantation becomes. So to promote xenotransplantation we have to stick to the difference and probably extend it, and at the same time make the most of the similarities.

Our way of thinking is not always changed by the use of arguments. We may want this to be the case, but the fact is that our way of thinking is often changed as a result of new technology. By changing our ways of life, technology also conveys new ideas and new ways of thinking. For this reason it is not far fetched to imagine that an extensive use of xenotransplantation may change our understanding of what a pig actually is. Through genetic engineering we may not just change the animals, but implicitly change our understanding of animals too. Animals have already, from a genetic point of view, become inconstant or contingent in a way never seen before. The genetic engineering we apply to the pigs today is of a minor kind. So it is still obvious to speak of a genetically altered pig as a pig. But these restrictions put on our genetic engineering are in no way necessary; they are minor just for the time being. So I think we must prepare ourselves for questions more far reaching than the questions we confront today. Questions I have in mind are questions such as: when is a genetically modified heart of a pig not a pig's heart any more? And when is a genetically altered pig not a pig any more? And if it isn't a pig, then what is it? Let us imagine that the demand for modified pig's hearts suddenly is satisfied. Is it possible to sell the surplus as 
pork, or is it cannibalism to eat hearts from these pigs? Is the consequence of xenotransplantation the establishment of a new category of living creatures, labelled replacement parts? And what about the human understanding of what it is to be a human being. Will this understanding be changed if we transform animals to an assembly of replacement parts?

These questions may seem a bit gothic or even bizarre, but the point is that they uncover possible consequences implied in the use of this kind of new technology. Abhorring from these questions will not do us any good.

\section{Final considerations}

Questions and arguments raised in this lecture are not conclusive with regard to xenotrans- plantation. I am not saying that xenotransplantation is to be abandoned. What I am saying is, that if we are to put xenotransplantation in action, we have to pay serious attention to the source animal welfare perspective. This may not be the most important perspective, but it is nevertheless one of several important perspectives. If we can't afford time and money to take this perspective into account, xenotransplantation may not be the way to human flourishing that we expect today.

\section{References}

1. Animal-to-human transplants: The ethics of xenotransplantation. Nuffield Council of Bioethics, UK, 1996. 